\title{
Multiple solutions of Kazdan-Warner equation on graphs in the negative case
}

\author{
Shuang Liu, Yunyan Yang ${ }^{1}$ \\ Department of Mathematics, Renmin University of China, Beijing 100872, P. R. China
}

\begin{abstract}
Let $G=(V, E)$ be a finite connected graph, and let $\kappa: V \rightarrow \mathbb{R}$ be a function such that $\int_{V} \kappa d \mu<0$. We consider the following Kazdan-Warner equation on $G$ :

$$
\Delta u+\kappa-K_{\lambda} e^{2 u}=0,
$$

where $K_{\lambda}=K+\lambda$ and $K: V \rightarrow \mathbb{R}$ is a non-constant function satisfying $\max _{x \in V} K(x)=0$ and $\lambda \in \mathbb{R}$. By a variational method, we prove that there exists a $\lambda^{*}>0$ such that when $\lambda \in\left(-\infty, \lambda^{*}\right]$ the above equation has solutions, and has no solution when $\lambda \geq \lambda^{*}$. In particular, it has only one solution if $\lambda \leq 0$; at least two distinct solutions if $0<\lambda<\lambda^{*}$; at least one solution if $\lambda=\lambda^{*}$. This result complements earlier work of Grigor'yan-Lin-Yang [7], and is viewed as a discrete analog of that of Ding-Liu [4] and Yang-Zhu [17] on manifolds.
\end{abstract}

Key words: Kazdan-Warnar problem on graph, variation problem on graph 2010 MSC: 35R02, 34B45

\section{Introduction}

Variational method is always a powerful tool in partial differential equations and geometric analysis. Recently, using this tool, Grigor'yan-Lin-Yang [7, 8, 9] obtained existence results for solutions to various partial differential equations on graphs. In particular, Kazdan-warnar equation was proposed on graphs in [7]. The Kazdan-Warner equation arises from the basic geometric problem on prescribing Gaussian curvature of Riemann surface, which systematically studied by Kazdan-Warner [12, 13]. On a closed Riemann surface $(\Sigma, g)$ with the Gaussian curvature $\kappa$, let $\widetilde{g}=e^{2 u} g$ be a smooth metric conformal to $g$ and $K$ be the Gaussian curvature with respect to $\widetilde{g}$. Then $u$ satisfies the equation

$$
\Delta_{g} u+\kappa-K e^{2 u}=0,
$$

where $\Delta_{g}$ denotes the Laplace-Beltrami operator with respect to the metric $g$. Let $v$ be a solution to $\Delta_{g} v=\bar{\kappa}-\kappa$ and $f=2(u-v)$, where $\bar{\kappa}$ is the averaged integral of $\kappa$. Then the above equation is transformed to

$$
\Delta_{g} f+2 \bar{\kappa}-\left(2 K e^{2 v}\right) e^{f}=0 .
$$

\footnotetext{
${ }^{1}$ Corresponding author

Email addresses: shuangliu@ruc.edu.cn (Shuang Liu), yunyanyang@ruc.edu.cn (Yunyan Yang) Preprint submitted to $* * *$ 
Hence, one can free (11) from the geometric situation, and just studies the equation

$$
\Delta_{g} f+c-h e^{f}=0,
$$

where $c$ is a constant and $h$ is a function. On graphs, it seems to be out of reach to resemble this topic in terms of Gaussian curvature. Therefore, in [7], the authors focused on the equation similar to the form of (2), namely the Kazdan-Warner equation on graph, and obtained the following: when $c=0$, it has a solution if and only if $h$ changes sign and the integral of $h$ is negative; when $c>0$, it has a solution if and only if $h$ is positive somewhere; when $c<0$, there is a threshold $c_{h}<0$ such that it has a solution if $c \in\left(c_{h}, 0\right)$, but it has no solution for any $c<c_{h}$. Later, Ge [5] found a solution in the critical case $c=c_{h}$. More recently Ge-Jiang [6] studied the Kazdan-Warner equation on infinite graphs and Keller-Schwarz [11] on canonically compactifiable graphs; Camilli-Marchi [3] extended the Kazdan-Warner equation on network; for other related works, we refer the readers to [10, 14].

Let us come back to a closed Riemann surface $(\Sigma, g)$, whose Euler characteristic is negative, or equivalently $\int_{\Sigma} \kappa d v_{g}<0$. Replacing $K$ by $K+\lambda$ in (1) with $K \leq 0, K \neq 0$, and $\lambda \in \mathbb{R}$, Ding-Liu [4] obtained the following conclusion by using a method of upper and lower solutions and a variational method: there exists a $\lambda^{*}>0$ such that if $\lambda \leq 0$, then (11) has a unique solution; if $0<\lambda<\lambda^{*}$, then (11) has at least two distinct solutions; if $\lambda=\lambda^{*}$, then (1) has at least one solution; if $\lambda>\lambda^{*}$, then (1) has no solution. Recently, this result was partly reproved by BorerGalimberti-Struwe [2] via a monotonicity technique due to Struwe [15, 16], and was extended to the case of conical metrics by Yang-Zhu [17].

Our aim is to extend results of Ding-Liu [4] to graphs. Let us recall some notations from graph theory. Throughout this paper, $G=(V, E)$ is assumed to be a finite connected graph. The edges on the graph are allowed to be weighted. Weights are given by a function $\omega: V \times V \rightarrow$ $[0, \infty)$, the edge $x y$ from $x$ to $y$ has weight $\omega_{x y}>0$. We assume this weight function is symmetric, $\omega_{x y}=\omega_{y x}$. Let $\mu: V \rightarrow \mathbb{R}^{+}$be a positive measure on the vertices of the $G$. Denote by $V^{\mathbb{R}}$ the space of real functions on $V$. and by $\ell_{\mu}^{p}=\left\{f \in V^{\mathbb{R}}: \sum_{x \in V} \mu(x)|f(x)|^{p}<\infty\right\}$, for any $1 \leq p<\infty$, the space of $\ell^{p}$ integrable functions on $V$ with respect to the measure $\mu$. For $p=\infty$, let $\ell^{\infty}=\left\{f \in V^{\mathbb{R}}: \sup _{x \in V}|f(x)|<\infty\right\}$ be the set of all bounded functions. As usual, we define the $\ell_{\mu}^{p}$ norm of $f \in \ell_{\mu}^{p}, 1 \leq p \leq \infty$, by

$$
\|f\|_{p}=\left(\sum_{x \in V} \mu(x)|f(x)|^{p}\right)^{1 / p}, 1 \leq p<\infty,\|f\|_{\infty}=\sup _{x \in V}|f(x)| .
$$

We define the Laplacian $\Delta: V^{\mathbb{R}} \rightarrow V^{\mathbb{R}}$ on $G$ by

$$
\Delta f(x)=\frac{1}{\mu(x)} \sum_{y \sim x} \omega_{x y}(f(x)-f(y)) .
$$

Given the weight $\omega$ on $E$, there are two typical choices of Laplacian as follows:

- $\mu(x)=\operatorname{deg}(x):=\sum_{y \sim x} \omega_{x y}$ for all $x \in V$, which is called the normalized graph Laplacian;

- $\mu(x) \equiv 1$ for all $x \in V$, which is the combinatorial graph Laplacian.

In this paper, we do not restrict $\mu(x)$ to the above two forms, but only require $\mu(x)>0$ for all $x \in V$. Note that the Laplace operator defined in (3) is the negative usual Laplace operator. The 
gradient form is defined by

$$
\begin{aligned}
2 \Gamma(f, g)(x) & =(f \cdot \Delta g+g \cdot \Delta f-\Delta(f \cdot g))(x) \\
& =\frac{1}{\mu(x)} \sum_{y \sim x} \omega_{x y}(f(x)-f(y))(g(x)-g(y)) .
\end{aligned}
$$

For the sake of simplicity, we write $\Gamma(f, f)=\Gamma(f)$. Sometimes we use the notation $\nabla f \nabla g=$ $\Gamma(f, g)$. The length of the gradient is denoted by

$$
|\nabla f|(x)=\sqrt{\Gamma(f)}(x) .
$$

From now on, we write $\int_{V} u d \mu=\sum_{x \in V} \mu(x) u(x)$. Define a Sobolev space with a norm on the graph $G$ by

$$
W^{1,2}(V)=\left\{u \in V^{\mathbb{R}}: \int_{V}\left(|\nabla u|^{2}+u^{2}\right) d \mu<+\infty\right\},
$$

and

$$
\|u\|_{W^{1,2}(V)}=\left(\int_{V}\left(|\nabla u|^{2}+u^{2}\right) d \mu\right)^{1 / 2}
$$

respectively. Since $G$ is a finite graph, we have that $W^{1,2}(V)$ is exactly $V^{\mathbb{R}}$, a finite dimensional linear space. This implies the following Sobolev embedding:

Lemma 1 ([7], Lemma 5). If $G$ is a finite graph, then the Sobolev space $W^{1,2}(V)$ is precompact. Namely, if $\left\{u_{j}\right\}$ is bounded in $W^{1,2}(V)$, then there exists some $u \in W^{1,2}(V)$ such that up to a subsequence, $u_{j} \rightarrow u$ in $W^{1,2}(V)$.

The Kazdan-Warner equation we are interested in this paper reads as

$$
\Delta u+\kappa-K_{\lambda} e^{2 u}=0 \quad \text { on } \quad V,
$$

where $\kappa \in V^{\mathbb{R}}$ is a function, and $K_{\lambda}=K+\lambda, \lambda \in \mathbb{R}, K \in V^{\mathbb{R}}$ is a function. Now we are ready to state our main results.

Theorem 2. Let $G=(V, E)$ be a finite graph, $\kappa$ and $K_{\lambda}$ be given as in (4) such that $\int_{V} \kappa d \mu<0$, $K \leq \max _{V} K=0$, and $K \neq 0$. Then there exists a $\lambda^{*} \in\left(0,-\min _{V} K\right)$ satisfying

1. if $\lambda \leq 0$, then (4) has a unique solution;

2. if $0<\lambda<\lambda^{*}$, then (4) has at least two distinct solutions;

3. if $\lambda=\lambda^{*}$, then (4) has at least one solution;

4. if $\lambda>\lambda^{*}$, then (4) has no solution;

Remark 1. The assertion of $\lambda^{*}<-\min _{V} K$ comes from the conclusion of Step 2 in Subsection 3.3.

Remark 2. Compared to the existence of solutions in the literature (see for example [7, 5]), the above results firstly reveal the multiple solution problem of Kazdan-Warner equation on graphs in the negative case.

The proof of Theorem 2 is based on the method of variation. It can be viewed as a discrete analog of the result of Ding-Liu [4]. The remaining part of this paper will be organized as follows: In Section 2] we give several preliminary lemmas for our use later; In Section 3] we finish the proof of Theorem 2 


\section{Preliminaries}

In this section, we provide discrete versions of the maximum principle, the Palais-Smale condition and the upper and lower solution principle. Note that $G=(V, E)$ is a finite connected graph.

\section{1. maximum principle}

To proceed, we need the following maximum principles, which are known for experts (see for examples [7, 8]). For readers' convenience, we include the detailed proofs here.

Lemma 3 (Weak maximum principle). For any constant $c>0$, if $u$ satisfies $\Delta u+c u \geq 0$, then $u \geq 0$ on $V$.

Proof. Let $u^{-}=\min \{u, 0\}$. For any $x \in V$, we claim that

$$
\Delta u^{-}(x)+c u^{-}(x) \geq 0
$$

from which, one has

$$
\int_{V} \Gamma\left(u^{-}\right) d \mu+c\left\|u^{-}\right\|_{\ell_{\mu}^{2}}^{2}=\left\langle u^{-}, \Delta u^{-}+c u^{-}\right\rangle \leq 0 .
$$

This leads to $u^{-} \equiv 0$ on $V$.

To prove this claim, we first consider the case $u(x) \geq 0$. Therefore, $c u^{-}(x)=0$ and

$$
\Delta u^{-}(x)=\frac{1}{\mu(x)} \sum_{y \sim x} \omega_{x y}\left(u^{-}(x)-u^{-}(y)\right)=-\frac{1}{\mu(x)} \sum_{y \sim x} \omega_{x y} u^{-}(y) \geq 0,
$$

due to $u^{-}(z) \leq 0$ for any $z \in V$. In the case $u(x)<0$, one has $c u^{-}(x)=c u(x)$ and thus

$$
\begin{aligned}
\Delta u^{-}(x)=\frac{1}{\mu(x)} \sum_{y \sim x} \omega_{x y}\left(u^{-}(x)-u^{-}(y)\right) & =\frac{1}{\mu(x)} \sum_{y \sim x} \omega_{x y}\left(u(x)-u^{-}(y)\right) \\
& \geq \frac{1}{\mu(x)} \sum_{y \sim x} \omega_{x y}(u(x)-u(y))=\Delta u(x) .
\end{aligned}
$$

It follows that $\Delta u^{-}(x)+c u^{-}(x) \geq \Delta u(x)+c u(x) \geq 0$, which confirms (5) and ends the proof of the lemma.

Lemma 4 (Strong maximum principle). Suppose that $u \geq 0$, and that $\Delta u+c u \geq 0$ for some constant $c>0$. If there exists $x_{0} \in V$ such that $u\left(x_{0}\right)=0$, then $u \equiv 0$ on $V$.

Proof. Let $x=x_{0}$, we have

$$
\frac{1}{\mu\left(x_{0}\right)} \sum_{y \sim x_{0}} \omega_{y x_{0}}\left(u\left(x_{0}\right)-u(y)\right)+c u\left(x_{0}\right) \geq 0,
$$

which implies

$$
\frac{1}{\mu\left(x_{0}\right)} \sum_{y \sim x_{0}} \omega_{y x_{0}} u(y) \leq 0 .
$$

Since $u \geq 0$ and $\omega_{y x_{0}}>0$ for all $y \sim x_{0}$, we obtain

$$
u(y)=0, \quad \text { for all } y \sim x_{0} .
$$

Therefore, $u \equiv 0$ on $V$ by the connectedness of $G$. 


\subsection{Palais-Smale condition}

We define a functional $E_{\lambda}: W^{1,2}(V) \rightarrow \mathbb{R}$ by

$$
E_{\lambda}(u)=\int_{V}\left(|\nabla u|^{2}+2 \kappa u-K_{\lambda} e^{2 u}\right) d \mu,
$$

where $\kappa$ and $K_{\lambda}$ are given as in the assumptions of Theorem 2, in particular $\int_{V} \kappa d \mu<0$. For any $\phi \in W^{1,2}(V)$, denote by $d E_{\lambda}(u)(\phi)$ the Frechet derivative of the functional, by $d^{k} E_{\lambda}(u)(\phi, \cdots, \phi)$ the Frechet derivative of order $k \geq 2$.

Lemma 5 (Palais-Smale condition). Suppose that $V_{\lambda}^{-}=\left\{x \in V: K_{\lambda}(x)<0\right\}$ is nonempty for some $\lambda \in \mathbb{R}$. Then $E_{\lambda}$ satisfies the $(P S)_{c}$ condition for all $c \in \mathbb{R}$, i.e. if $\left(u_{j}\right)$ is a sequence of functions in $W^{1,2}(V)$ such that $E_{\lambda}\left(u_{j}\right) \rightarrow c$ and $d E_{\lambda}\left(u_{j}\right)(\phi) \rightarrow 0$ for all $\phi \in W^{1,2}(V)$ as $j \rightarrow \infty$, then there exists some $u_{0} \in W^{1,2}(V)$ satisfying $u_{j} \rightarrow u_{0}$ in $W^{1,2}(V)$.

Proof. Let $\left(u_{j}\right)$ be a function sequence such that $E_{\lambda}\left(u_{j}\right) \rightarrow c$ and $d E_{\lambda}\left(u_{j}\right)(\phi) \rightarrow 0$, or equivalently

$$
\begin{gathered}
\int_{V}\left(\left|\nabla u_{j}\right|^{2}+2 \kappa u_{j}-K_{\lambda} e^{2 u_{j}}\right) d \mu=c+o_{j}(1), \\
\int_{V}\left(\nabla u_{j} \nabla \phi+\kappa \phi-K_{\lambda} e^{2 u_{j}} \phi\right) d \mu=o_{j}(1)\|\phi\|_{W^{1,2}(V)}, \quad \forall \phi \in W^{1,2}(V),
\end{gathered}
$$

where $o_{j}(1) \rightarrow 0$ as $j \rightarrow \infty$.

Let $\phi \equiv 1$ in $(77$, one has

$$
\int_{V}\left(\kappa-K_{\lambda} e^{2 u_{j}}\right) d \mu=o_{j}(1) \mu(V)^{1 / 2}
$$

which implies

$$
\int_{V} K_{\lambda} e^{2 u_{j}} d \mu=\int_{V} \kappa d \mu+o_{j}(1)
$$

Inserting (8) into (6), we obtain

$$
\int_{V}\left(\left|\nabla u_{j}\right|^{2}+2 \kappa u_{j}\right) d \mu=\int_{V} \kappa d \mu+c+o_{j}(1) .
$$

We now claim that $u_{j}$ is bounded in $\ell_{\mu}^{2}$. Suppose not, there holds $\left\|u_{j}\right\|_{\ell_{\mu}^{2}} \rightarrow \infty$. We set $v_{j}=\frac{u_{j}}{\left\|u_{j}\right\|_{\ell_{\mu}}}$. By the Cauchy-Schwarz inequality, one has

$$
\int_{V} \kappa \frac{u_{j}}{\left\|u_{j}\right\|_{\ell_{\mu}^{2}}^{2}} d \mu=o_{j}(1)
$$

This together with (9) leads to

$$
\int_{V}\left|\nabla v_{j}\right|^{2} d \mu=o_{j}(1) .
$$

Hence, $v_{j}$ is bounded in $W^{1,2}(V)$. In view of Lemma 1 and $(10), v_{j} \rightarrow \gamma$ in $W^{1,2}(V)$ for some constant $\gamma$. Here and in the sequel, we do not distinguish sequence and subsequence. Since $\left\|v_{j}\right\|_{\ell_{\mu}^{2}}=1$, we have $\gamma \neq 0$. It follows from (9) that

$$
\int_{V} \kappa v_{j} d \mu \leq o_{j}(1) .
$$


Passing to the limit $j \rightarrow \infty$ in the above inequality, we conclude that $\gamma \geq 0$ since $\int_{V} \kappa d \mu<0$. Therefore $\gamma>0$.

On the other hand, for any $x \in V_{\lambda}^{-}$, if there exists $N \in \mathbb{N}$, if $j>N$ such that $u_{j}(x) \leq 0$, then $\lim _{j \rightarrow \infty} v_{j}(x) \leq 0$, which contradicts $\gamma>0$ and confirms our claim. If not, let $x_{*} \in V_{\lambda}^{-}$, due to the finiteness of $V$, we can choose a subsequence $\left\{j_{k}\right\}_{k=0}^{\infty}$ such that $u_{j_{k}}\left(x_{*}\right)>0$. Set

$$
\phi(x)= \begin{cases}u_{j_{k}}\left(x_{*}\right), & x=x_{*} \\ 0, & x \neq x_{*}\end{cases}
$$

Then

$$
\|\phi\|_{W^{1,2}(V)}^{2}=2 \sum_{y \sim x_{*}} \omega_{x_{*} y} u_{j_{k}}^{2}\left(x_{*}\right)+\mu\left(x_{*}\right) u_{j_{k}}^{2}\left(x_{*}\right)=\left(2 \operatorname{deg}\left(x_{*}\right)+\mu\left(x_{*}\right)\right) u_{j_{k}}^{2}\left(x_{*}\right) .
$$

Substituting it into (7), we have

$$
\Delta u_{j_{k}}\left(x_{*}\right)+\kappa\left(x_{*}\right)-K_{\lambda}\left(x_{*}\right) e^{2 u_{j_{k}}\left(x_{*}\right)} \leq C^{\prime} .
$$

Since $v_{j} \rightarrow \gamma,\left\|u_{j}\right\|_{\ell_{\mu}^{2}} \rightarrow+\infty$ and $V$ has finite points, we conclude

$$
u_{j}=\left(\gamma+o_{j}(1)\right)\left\|u_{j}\right\|_{\ell_{\mu}^{2}} \quad \text { uniformly on } \quad V .
$$

This together with (11) leads to

$$
\left\|u_{j_{k}}\right\|_{\ell_{\mu}^{2}}^{2} o_{j_{k}}(1)+\kappa\left(x_{*}\right)-K_{\lambda}\left(x_{*}\right) e^{2\left(\gamma+o_{j_{k}}(1)\right)\left\|u_{j_{k}}\right\|_{\rho_{\mu}}^{2}} \leq C^{\prime}
$$

which is impossible since $K_{\lambda}\left(x_{*}\right)<0$. Then our claim follows immediately.

Since $u_{j}$ is bounded in $\ell_{\mu}^{2}$, we have $u_{j}$ is bounded in $W^{1,2}(V)$ due to the finiteness of $V$. Therefore, by Lemma 1 there exists some $u_{0} \in W^{1,2}(V)$ such that up to subsequence, $u_{j} \rightarrow u_{0}$ in $W^{1,2}(V)$

\subsection{Upper and lower solutions principle}

Let $f: V \times \mathbb{R} \rightarrow \mathbb{R}$ be a function, and $f$ is smooth with respect to the second variable. We say that $u \in V^{\mathbb{R}}$ is an upper (lower) solution to the following equation

$$
\Delta u(x)+f(x, u(x))=0, \quad x \in V,
$$

if $u$ satisfies $\Delta u(x)+f(x, u(x)) \geq(\leq) 0$ for any $x \in V$. We generalize ([7], Lemma 8) to the following:

Lemma 6. Suppose that $\varphi, \psi$ are lower and upper solution to 12 respectively with $\varphi \leq \psi$ on $V$. Then (12) has a solution $u$ with $\varphi \leq u \leq \psi$ on $V$.

Proof. This is a discrete version of the argument of Kazdan-Warner ([12], Lemma 9.3), and the method of proof carries over to the setting of graphs.

Since the graph is finite, there exists a constant $A$ such that $-A \leq \varphi \leq \psi \leq A$. One can find a sufficient large constant $c$ such that $F(x, t)=c t-f(x, t)$ is increasing with respect to $t \in[-A, A]$ for any fixed $x \in V$. We define an operator $L u=\Delta u+c u$, and $L$ is a compact operator and $\operatorname{Ker}(L)=\operatorname{span}\{1\}$ due to the finiteness of the graph. Hence, we can define $\varphi_{j+1}, \psi_{j+1}$ inductively as the unique solution to

$$
\varphi_{0}=\varphi, L \varphi_{j+1}(x)=c \varphi_{j}(x)-f\left(x, \varphi_{j}(x)\right), \quad \forall j \geq 0, x \in V,
$$




$$
\psi_{0}=\psi, L \psi_{j+1}(x)=c \psi_{j}(x)-f\left(x, \psi_{j}(x)\right), \quad \forall j \geq 0, x \in V
$$

respectively. Combining with the definition of upper (lower) solution and the monotonicity of $F(x, t)$ with respect to $t$, we obtain

$$
L \varphi_{0}(x) \leq L \varphi_{1}(x)=F(x, \varphi(x)) \leq F(x, \psi(x))=L \psi_{1}(x) \leq L \psi(x), \quad x \in V .
$$

Then the weak maximum principle (see Lemma3) yields that

$$
\varphi \leq \varphi_{1} \leq \psi_{1} \leq \psi
$$

Moreover, it turns out that $\varphi_{1}$ and $\psi_{1}$ are lower and upper solution to (12) respectively. By induction, we have

$$
\varphi \leq \varphi_{j} \leq \varphi_{j+1} \leq \psi_{j+1} \leq \psi_{j} \leq \psi, \quad j=1,2, \cdots .
$$

Since $V$ is finite, it is easy to see that up to a subsequence, $\varphi_{j} \rightarrow u_{1}, \psi_{j} \rightarrow u_{2}$ uniformly on $V$, and $u=u_{1}$ or $u_{2}$ is a solution to (12) with $\varphi \leq u \leq \psi$ on $V$.

\section{Proof of Theorem 2}

3.1. Unique solution in the case $\lambda \leq 0$.

Claim 1. $E_{\lambda}$ is strictly convex on $W^{1,2}(V)$.

Proof. We only need to show that there exists some constant $C>0$ such that

$$
d^{2} E_{\lambda}(u)(h, h) \geq C\|h\|_{W^{1,2}(V)}^{2}, \quad \forall u, h \in W^{1,2}(V) .
$$

Suppose not, there would be a function $u \in W^{1,2}(V)$ and a function sequence $h_{j} \in W^{1,2}(V)$ such that $\left\|h_{j}\right\|_{W^{1,2}(V)}=1$ for all $j$ and $d^{2} E_{\lambda}(u)\left(h_{j}, h_{j}\right) \rightarrow 0$ as $j \rightarrow \infty$. From Lemma1 there exists $h_{\infty} \in W^{1,2}(V)$, such that up to a subsequence, $h_{j} \rightarrow h_{\infty}$ as $j \rightarrow \infty$ in $W^{1,2}(V)$. Since

$$
d^{2} E_{\lambda}(u)\left(h_{j}, h_{j}\right)=2 \int_{V}\left(\left|\nabla h_{j}\right|^{2}-2 K_{\lambda} e^{2 u} h_{j}^{2}\right) d \mu,
$$

and $K_{\lambda} \leq 0$, it follows that $\int_{V}\left|\nabla h_{j}\right|^{2} d \mu \rightarrow 0$ and $\int_{V} K_{\lambda} e^{2 u} h_{j}^{2} d \mu \rightarrow 0$, which lead to $h_{\infty} \equiv c$ for some constant $c$, and moreover

$$
c^{2} \int_{V} K_{\lambda} e^{2 u} d \mu=\lim _{j \rightarrow \infty} \int_{V} K_{\lambda} e^{2 u} h_{j}^{2} d \mu=0 .
$$

It is easily seen that $\int_{V} K_{\lambda} e^{2 u} d \mu<0$ by $K \not \equiv 0$, thus $c=0$. This contradicts

$$
\left\|h_{\infty}\right\|_{W^{1,2}(V)}=\lim _{j \rightarrow \infty}\left\|h_{j}\right\|_{W^{1,2}(V)}=1 .
$$

Hence (13) holds.

Claim 2. For any $\varepsilon>0$, there exist constants $C, C(\varepsilon)>0$ such that

$$
E_{\lambda}(u) \geq(C-2 \varepsilon)\|u\|_{W^{1,2}(V)}^{2}-2 C(\varepsilon) .
$$


Proof. By Young's inequality, for any $\varepsilon>0$, there exists a constant $C(\varepsilon)>0$ such that

$$
\left|\int_{V} \kappa u d \mu\right| \leq \varepsilon\|u\|_{W^{1,2}(V)}^{2}+C(\varepsilon)
$$

Thus, it is sufficient to find some constant $C>0$ such that for all $u \in W^{1,2}(V)$

$$
\int_{V}\left(|\nabla u|^{2}-K_{\lambda} e^{2 u}\right) d \mu \geq C\|u\|_{W^{1,2}(V)}^{2} .
$$

Suppose not, there would exist a sequence of functions $u_{j}$ satisfying

$$
\int_{V}\left(\left|\nabla u_{j}\right|^{2}+u_{j}^{2}\right) d \mu=1, \quad \int_{V}\left(\left|\nabla u_{j}\right|^{2}-K_{\lambda} e^{2 u_{j}}\right) d \mu=o_{j}(1) .
$$

Clearly, $u_{j}$ is bounded in $W^{1,2}(V)$, it follows from Lemma 1 that there exists some function $u \in W^{1,2}(V)$ such that up to a subsequence, $u_{j} \rightarrow u$ in $W^{1,2}(V)$ as $j \rightarrow \infty$. Due to $K_{\lambda} \not \equiv 0$ and $K_{\lambda} \leq 0$, we have

$$
0<\int_{V}\left(|\nabla u|^{2}-K_{\lambda} e^{2 u}\right) d \mu=\lim _{j \rightarrow \infty} \int_{V}\left(\left|\nabla u_{j}\right|^{2}-K_{\lambda} e^{2 u_{j}}\right) d \mu=0,
$$

which gets a contradiction.

Proof of (1) in Theorem 2 It is a consequence of Claim 1 and Claim 2 Precisely we denote $\Lambda=\inf _{u \in W^{1,2}(V)} E_{\lambda}(u)$. By Claim 2, we see that $\Lambda$ is a definite real number. Take a function sequence $u_{j} \in W^{1,2}(V)$ such that $E_{\lambda}\left(u_{j}\right) \rightarrow \Lambda$ as $j \rightarrow \infty$. Applying Claim 2 we have that $u_{j}$ is bounded in $W^{1,2}(V)$. Then, in view of Lemma1 there exists a subsequence of $u_{j}$ (still denoted by $u_{j}$ ) and a function $u_{0} \in W^{1,2}(V)$ such that $u_{j} \rightarrow u_{0}$ in $W^{1,2}(V)$. Obviously $E_{\lambda}\left(u_{0}\right)=\Lambda$, and thus $u_{0}$ is a critical point of $E_{\lambda}$. We also need to explain why $E_{\lambda}$ has only one critical point. For otherwise, we assume $u^{*}$ is another critical point of $E_{\lambda}$. Note that $d E_{\lambda}\left(u_{0}\right)=d E_{\lambda}\left(u^{*}\right)=0$. It follows from Claim 1 particularly from (13), that

$$
\begin{aligned}
E_{\lambda}\left(u_{0}\right) & =E_{\lambda}\left(u^{*}\right)+d E_{\lambda}\left(u^{*}\right)\left(u_{0}-u^{*}\right)+\frac{1}{2} d^{2} E_{\lambda}(\xi)\left(u_{0}-u^{*}, u_{0}-u^{*}\right) \\
& \geq E_{\lambda}\left(u^{*}\right)+C\left\|u_{0}-u^{*}\right\|_{W^{1,2}(V)}^{2}
\end{aligned}
$$

for some positive constant $C$, where $\xi$ is a function lies between $u^{*}$ and $u_{0}$. Hence we have $E_{\lambda}\left(u_{0}\right)>E_{\lambda}\left(u^{*}\right)$, contradicting the fact that $E_{\lambda}\left(u_{0}\right)=\Lambda$. This implies the uniqueness of the critical point of $E_{\lambda}$.

\subsection{Multiplicity of solutions for $0<\lambda<\lambda^{*}$.}

Fixing $\lambda \in\left(0, \lambda^{*}\right)$, we will seek two different solutions of (4). One is a strict local minimum of the functional $E_{\lambda}$, and the other is from mountain-pass theorem. We firstly prove the existence of $\lambda^{*}$. Consider the case $\lambda=0$ in the equation (4) as follows

$$
\Delta u+\kappa-K e^{2 u}=0 .
$$

For the solution $u_{0}$ of (14), the linearized equation of (14) at $u_{0}$

$$
\begin{gathered}
\Delta v-2 K e^{2 u_{0}} v=0 \\
8
\end{gathered}
$$


has only a trivial solution $v \equiv 0$, since $K \leq 0$ and $K \not \equiv 0$. Indeed,

$$
0 \leq \int_{V}|\nabla v|^{2} d \mu=\int_{V} v \Delta v d \mu=2 \int_{V} K e^{2 u_{0}} v^{2} d \mu \leq 0,
$$

which implies $v(x)=0$ when $K(x)<0$. In the case $K(x)=0$, we have $\Delta v(x)=0$. Thus

$$
\int_{V}|\nabla v|^{2} d \mu=\int_{V} v \Delta v d \mu=0 .
$$

It follows that $v$ is a constant function and hence $v \equiv 0$. By the implicit function theorem, there exists a small enough $s>0$ such that the equation (4) has a solution for any $\lambda \in(0, s)$. Indeed, let $u=t v+u_{0}, v \neq \equiv$ on $V$, we consider $G(\lambda, t)=\Delta u+\kappa-K_{\lambda} e^{u}$. It is easy to see that $G(0,0)=0$, $G(\lambda, t)$ and $\partial_{t} G(\lambda, t)=\Delta v-2 v(K+\lambda) e^{2\left(u_{0}+t v\right)}$ are continuous on any domain $D \subset \mathbb{R}^{2}$, Furthermore, $\partial_{t} G(0,0)=\Delta v-2 K e^{2 u_{0}} v \not \equiv 0$ unless $v \equiv 0$ on $V$. Therefore, by the implicit function theorem, there exists $s>0$ such that $t=g(\lambda)$ and $G(\lambda, g(\lambda))=0$ for any $\lambda \in(0, s)$. In other words, $u_{\lambda}=g(\lambda) v+u_{0}, \forall \lambda \in(0, s)$ is the solution of (4). Define

$$
\lambda^{*}=\sup \{s: \text { the equation (4) has a solution for any } \lambda \in(0, s)\} .
$$

One can see that $\lambda^{*} \leq-\min _{V} K$. For otherwise, $K_{\lambda}=K+\lambda>0$ for some $\lambda<\lambda^{*}$. Adding up the equation (4) for all $x \in V$, we have

$$
0>\int_{V} \kappa d \mu=\int_{V} K_{\lambda} e^{2 u} d \mu>0
$$

which is impossible. In conclusion, we have $0<\lambda^{*} \leq-\min _{V} K$.

Proof of (2) in Theorem 2 We separate the proof into the following three steps.

Step 1. The existence of the upper and lower solution of (4).

Take $\lambda_{1}$ with $\lambda<\lambda_{1}<\lambda^{*}$, let $u_{\lambda_{1}}$ be a solution of (4) at $\lambda_{1}$. It is easily seen that $\psi=u_{\lambda_{1}}$ is a strict upper solution of (4) at $\lambda$, namely

$$
\Delta \psi+\kappa-K_{\lambda} e^{2 \psi}>0
$$

Let $v$ be the solution to the following equation

$$
\Delta v=-\kappa+\frac{1}{\mu(V)} \int_{V} \kappa d \mu .
$$

The existence of solution to [15) was proved in [7]. Set $\varphi=v-s$, where $s$ is a sufficiently large constant such that $\varphi<\psi$ on $V$ and

$$
\Delta \varphi+\kappa-K_{\lambda} e^{2 \varphi}=\frac{1}{\mu(V)} \int_{V} \kappa d \mu-K_{\lambda} e^{2 v-2 s}<0
$$

since $\int_{V} \kappa d \mu<0$. Therefore, $\varphi$ is a strict lower solution of (4). Let $[\varphi, \psi]$ be the order interval defined by

$$
[\varphi, \psi]=\left\{u \in V^{\mathbb{R}}: \varphi \leq u \leq \psi \text { on } V\right\} .
$$

The upper and lower-solution method (Lemma 6) asserts that (4) has a solution $u_{\lambda} \in[\varphi, \psi]$ on $V$.

Step 2. $u_{\lambda}$ can be chosen as a strict local minimum of $E_{\lambda}$. 
Let $f_{\lambda}(x, t)=c t-\kappa(x)+K_{\lambda}(x) e^{2 t}$, where $c$ is sufficiently large such that $f_{\lambda}(x, t)$ is increasing in $t \in[-A, A], A$ is a constant such that $-A \leq \varphi<\psi \leq A$ on $V$. Let $F_{\lambda}(x, u(x))=\int_{0}^{u(x)} f_{\lambda}(x, t) d t$. It is easy to rewrite $E_{\lambda}(u)$ as

$$
E_{\lambda}(u)=\int_{V}\left(|\nabla u|^{2}+c u^{2}\right) d \mu-2 \sum_{x \in V} \mu(x) F_{\lambda}(x, u(x))-\int_{V} K_{\lambda} d \mu .
$$

It is obvious that $E_{\lambda}$ is bounded from below on $[\varphi, \psi]$. Therefore, we denote

$$
a:=\inf _{u \in[\varphi, \psi]} E_{\lambda}(u)
$$

Taking a function sequence $u_{j} \subset[\varphi, \psi]$ such that $E_{\lambda}\left(u_{j}\right) \rightarrow a$ as $j \rightarrow \infty$. From it, we can get that $u_{j}$ is bounded in $W^{1,2}(V)$, and thus up to subsequence, $u_{j}$ converges to some $u_{\lambda}$ in $W^{1,2}(V)$ and $\ell_{\mu}^{q}$ for any $q \geq 1$, and $e^{2 u_{j}}$ converges to $e^{2 u_{\lambda}}$ in $\ell_{\mu}^{1}$. Hence

$$
E_{\lambda}\left(u_{\lambda}\right)=\inf _{u \in[\varphi, \psi]} E_{\lambda}(u) .
$$

As a consequence, $u_{\lambda}$ satisfies the Euler-Lagrange equation

$$
\Delta u_{\lambda}(x)+c u_{\lambda}(x)=f_{\lambda}\left(x, u_{\lambda}(x)\right) .
$$

From it, one can conclude that

$$
\varphi(x)<u_{\lambda}(x)<\psi(x), \forall x \in V .
$$

Indeed, noting that $f_{\lambda}(x, t)$ is increasing with respect to $t \in[-A, A]$, we have

$$
\Delta \varphi(x)+c \varphi(x) \leq f_{\lambda}(x, \varphi(x)) \leq f_{\lambda}(x, \psi(x)) \leq \Delta \psi(x)+c \psi(x) .
$$

One can conclude (16) by the strong maximum principle (Lemma44), and the fact $\varphi<\psi$. For any $h \in W^{1,2}(V)$, we define a function $\eta(t)=E_{\lambda}\left(u_{\lambda}+t h\right), t \in \mathbb{R}$. There holds $\varphi \leq u_{\lambda}+t h \leq \psi$ for sufficiently small $|t|$. Since $u_{\lambda}$ is a minimum of $E_{\lambda}$ on $(\varphi, \psi)$, we have $\eta^{\prime}(0)=d E_{\lambda}\left(u_{\lambda}\right)(h)=0$ and $\eta^{\prime \prime}(0)=d^{2} E_{\lambda}\left(u_{\lambda}\right)(h, h) \geq 0$. Furthermore, there exists a constant $C>0$ such that

$$
d^{2} E_{\lambda}\left(u_{\lambda}\right)(h, h) \geq C\|h\|_{W^{1,2}(V)}, \quad \forall h \in W^{1,2}(V),
$$

which implies $u_{\lambda}$ is strict local minimum of $E_{\lambda}$ on $W^{1,2}(V)$. It remains to prove 177). We first denote

$$
\theta:=\inf _{\|h\|_{W 1,2(V)}=1} d^{2} E_{\lambda}\left(u_{\lambda}\right)(h, h),
$$

which is nonnegative. It is sufficient to prove $\theta>0$, (17) follows. Suppose $\theta=0$, we claim that there exists some $\tilde{h}$ with $\|\tilde{h}\|_{W^{1,2}(V)}=1$ such that $d^{2} E_{\lambda}\left(u_{\lambda}\right)(\tilde{h}, \tilde{h})=0$. To see this, let $h_{j}$ be a function sequence satisfying $\left\|h_{j}\right\|_{W^{1,2}(V)}=1$ for all $j$ and $d^{2} E_{\lambda}\left(u_{\lambda}\right)\left(h_{j}, h_{j}\right) \rightarrow 0$ as $j \rightarrow \infty$. Up to subsequence, $h_{j} \rightarrow \tilde{h}$ in $W^{1,2}(V)$ from Lemma 1 and confirms our claim. To put it another way, the functional $v \mapsto d^{2} E_{\lambda}\left(u_{\lambda}\right)(v, v)$ attains its minimum at $v=\tilde{h}$, it follows that $d^{2} E_{\lambda}\left(u_{\lambda}\right)(\tilde{h}, v)=0$ for all $v \in W^{1,2}(V)$. Hence, $\tilde{h}$ is a solution of the following equation

$$
\Delta h=2 K_{\lambda} e^{2 u_{\lambda}} h, \quad \lambda \in\left(0, \lambda^{*}\right) .
$$


It is easy to see that $\tilde{h}$ is not a constant. For otherwise $(18)$ yields

$$
0>\int_{V} \kappa d \mu=\int_{V} K_{\lambda} e^{2 u_{\lambda}} d \mu=0,
$$

which is impossible. Multiplying (18) by $\tilde{h}^{3}$, we obtain

$$
\begin{aligned}
d^{4} E_{\lambda}\left(u_{\lambda}\right)(\tilde{h}, \tilde{h}, \tilde{h}, \tilde{h}) & =-16 \int_{V} K_{\lambda} e^{2 u_{\lambda}} \tilde{h}^{4} d \mu \\
& =-8 \int_{V} \tilde{h}^{3} \Delta \tilde{h} d \mu \\
& =-8 \sum_{x, y \in V} \omega_{x y}\left(\tilde{h}^{3}(x)-\tilde{h}^{3}(y)\right)(\tilde{h}(x)-\tilde{h}(y)) \\
& =-8 \sum_{x, y \in V} \omega_{x y}\left(\tilde{h}^{2}(x)+\tilde{h}(x) \tilde{h}(y)+\tilde{h}^{2}(y)\right)(\tilde{h}(x)-\tilde{h}(y))^{2}<0 .
\end{aligned}
$$

The last inequality is due to the fact $a^{2}+a b+b^{2}>0$ for any $a, b \in \mathbb{R}$ satisfying $a b \neq 0$. Since $d^{2} E_{\lambda}\left(u_{\lambda}+t \tilde{h}\right)(\tilde{h}, \tilde{h})$ attains its minimum at $t=0$, we have $d^{3} E_{\lambda}\left(u_{\lambda}\right)(\tilde{h}, \tilde{h}, \tilde{h})=0$, which together with $d E_{\lambda}\left(u_{\lambda}\right)(\tilde{h})=0$ and $d^{2} E_{\lambda}\left(u_{\lambda}\right)(\tilde{h}, \tilde{h})=0$ leads to

$$
E_{\lambda}\left(u_{\lambda}+\epsilon \tilde{h}\right)=E_{\lambda}\left(u_{\lambda}\right)+\frac{\epsilon^{4}}{24} d^{4} E_{\lambda}\left(u_{\lambda}\right)(\tilde{h}, \tilde{h}, \tilde{h}, \tilde{h})+0\left(\epsilon^{5}\right)<E_{\lambda}\left(u_{\lambda}\right)
$$

for small $\epsilon>0$. Let $\epsilon$ small enough such that $\varphi \leq u_{\lambda}+\epsilon \tilde{h} \leq \psi$, thus by [19],

$$
E_{\lambda}\left(u_{\lambda}+\epsilon \tilde{h}\right)<E_{\lambda}\left(u_{\lambda}\right),
$$

which contradicts the fact that $u_{\lambda}$ is the minimum of $E_{\lambda}$ on $[\varphi, \psi]$. Therefore $\theta>0$, which concludes (17).

Step 3. The second solution of (4) is given by the mountain-pass theorem.

We shall use the mountain-pass theorem due to Ambrosetti and Rabinowitz [1], which reads as follows: Let $(X,\|\cdot\|)$ be a Banach space, $J \in C^{1}(X, \mathbb{R}), e_{0}, e \in X$ and $r>0$ be such that $\left\|e-e_{0}\right\|>r$ and

$$
b:=\inf _{\left\|u-e_{0}\right\|=r} J(u)>J\left(e_{0}\right) \geq J(e) .
$$

If J satisfies the $(P S)_{c}$ condition with $c:=\inf _{\gamma \in \Gamma} \max _{t \in[0,1]} J(\gamma(t))$, where

$$
\Gamma:=\left\{\gamma \in C([0,1], X): \gamma(0)=e_{0}, \gamma(1)=e\right\},
$$

then $c$ is a critical value of $J$. In our case, $W^{1,2}(V)$ is a Banach space, and $E_{\lambda}: W^{1,2}(V) \rightarrow \mathbb{R}$ is a smooth functional.

Since $u_{\lambda}$ is a strict local minimum of $E_{\lambda}$ on $W^{1,2}(V)$, there exists a small enough number $r>0$ such that

$$
\inf _{\left\|u-u_{\lambda}\right\|_{W^{1,2},(V)}=r} E_{\lambda}(u)>E_{\lambda}\left(u_{\lambda}\right) .
$$

Moreover, for any $\lambda>0, E_{\lambda}$ has no lower bound on $W^{1,2}(V)$, namely, there exists $v \in W^{1,2}(V)$ such that

$$
E_{\lambda}(v)<E_{\lambda}\left(u_{\lambda}\right), \quad\left\|v-u_{\lambda}\right\|_{W^{1,2}(V)}>r
$$


To see this, we set $V_{\varepsilon}=\left\{x \in V: K_{\lambda}(x)>\varepsilon\right\}$ for small $\varepsilon>0$. Note that $V_{\varepsilon}$ is nonempty since $\max _{V} K=0$ and $\lambda>0$. Let $f \in W^{1,2}(V)$ be a function which equals to 1 in $V_{\varepsilon}$ and vanishes on $V / V_{\varepsilon}$, then

$$
\begin{aligned}
E_{\lambda}(t f) & =t^{2} \int_{V}|\nabla f|^{2} d \mu+t \int_{V_{\varepsilon}} \kappa d \mu-\int_{V_{\varepsilon}} K_{\lambda} e^{2 t} d \mu-\int_{V / V_{\varepsilon}} K_{\lambda} d \mu \\
& \leq A t^{2}+B t+C-\varepsilon \mu\left(V_{\varepsilon}\right) e^{2 t} \rightarrow-\infty, \quad t \rightarrow+\infty .
\end{aligned}
$$

In view of 201, (21) and Lemma 5, the mountain pass theorem of Ambrosetti and Rabinowitz gives another critical point $u^{\lambda}$ of $E_{\lambda}$ other than $u_{\lambda}$. In particular,

$$
E_{\lambda}\left(u^{\lambda}\right)=\min _{\gamma \in \Gamma} \max _{u \in \gamma} E_{\lambda}(u),
$$

where $\Gamma=\left\{\gamma \in C\left([0,1], W^{1,2}(V)\right): \gamma(0)=u_{\lambda}, \gamma(1)=v\right\}$, and $d E_{\lambda}\left(u^{\lambda}\right)(h)=0$ for any $h \in$ $W^{1,2}(V)$.

\subsection{Solvability at $\lambda^{*}$.}

For any $\lambda, 0<\lambda<\lambda^{*}$, let $u_{\lambda}$ be the local minimum of $E_{\lambda}$ obtained in the previous subsection. That is, $u_{\lambda}$ is the solution of (4), and

$$
d^{2} E_{\lambda}\left(u_{\lambda}\right)(h, h)=2 \int_{V}\left(|\nabla h|^{2}-2 K_{\lambda} e^{2 u_{\lambda}} h^{2}\right) d \mu \geq 0, \quad \forall h \in W^{1,2}(V) .
$$

Proof of (3) in Theorem 2 The crucial point in this proof is to show that $u_{\lambda}$ is uniformly bounded in $W^{1,2}(V)$ as $\lambda \rightarrow \lambda^{*}$. If it is true, then up to subsequence, $u_{\lambda}$ converges to some $u$ in $W^{1,2}(V)$, and $u$ is the solution of

$$
\Delta u+\kappa-K_{\lambda^{*}} e^{2 u}=0 .
$$

Hence, we aim to prove the $W^{1,2}(V)$ boundedness of $u_{\lambda}$. To this end, we divide the proof into the following three steps.

Step 1. There exists a constant $C>0$ such that

$$
u_{\lambda} \geq-C \text { on } V
$$

uniformly for any $0<\lambda<\lambda^{*}$.

Let $v$ satisfy (15), and $\varphi_{s}=v-s$ for $s>0$. Then for sufficient large $s$, say $s \geq s_{0}, \varphi_{s}$ is a continuous family with respect to $s$ of strict lower solution of (4) at $\lambda=0$, i.e.

$$
\Delta \varphi_{s}+\kappa-K e^{2 \varphi_{s}}<0 .
$$

It is clear that $\varphi_{s}$ is also a strict lower solution of (4) at $\lambda \in\left(0, \lambda^{*}\right)$ for any $s \in\left[s_{0}, \infty\right)$. Now, we prove that $u_{\lambda} \geq \varphi_{s_{0}}$, and thus (23) holds. For otherwise, we can find for some $s \in\left(s_{0}, \infty\right)$

$$
u_{\lambda} \geq \varphi_{s} \quad \text { on } V, \quad \text { and } u_{\lambda}(\tilde{x})=\varphi_{s}(\tilde{x}) \text { for some } \tilde{x} \in V .
$$

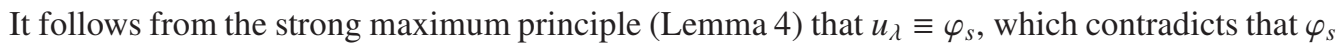
is strict low solution of (4) at $\lambda \in\left[0, \lambda^{*}\right)$.

Step 2. The set $V_{\lambda^{*}}^{-}=\left\{x \in V: K_{\lambda^{*}}(x)<0\right\}$ is not empty. 
From the case (1) in Theorem 2 , there exists unique solution $w_{0}$ of the equation

$$
\Delta w+\kappa+e^{2 w}=0 .
$$

Together with the solution $u_{\lambda}$ to the equation (4) at $\lambda$, and let $v_{\lambda}=u_{\lambda}-w_{0}$, we have

$$
\Delta v_{\lambda}-K_{\lambda} e^{2 u_{\lambda}}-e^{2 w_{0}}=0 .
$$

Multiplying the above equation by $e^{-2 v_{\lambda}}$ and intergrading by parts, one has

$$
\int_{V} K_{\lambda} e^{w_{0}} d \mu=\int_{V} e^{-2 v_{\lambda}} \Delta v_{\lambda} d \mu-\int_{V} e^{-2\left(u_{\lambda}-2 w_{0}\right)} d \mu \leq 0 .
$$

Indeed, by Green formula

$$
\int_{V} e^{-2 v_{\lambda}} \Delta v_{\lambda} d \mu=\sum_{x, y \in V} \omega_{x y}\left(v_{\lambda}(x)-v_{\lambda}(y)\right)\left(e^{-2 v_{\lambda}(x)}-e^{-2 v_{\lambda}(y)}\right) \leq 0
$$

since $\left(v_{\lambda}(x)-v_{\lambda}(y)\right)\left(e^{-2 v_{\lambda}(x)}-e^{-2 v_{\lambda}(y)}\right) \leq 0$ for any $x, y \in V$. Therefore

$$
\int_{V} K_{\lambda^{*}} e^{w_{0}} d \mu=\lim _{\lambda \rightarrow \lambda^{*}} \int_{V} K_{\lambda} e^{w_{0}} d \mu \leq 0
$$

Suppose that $K_{\lambda^{*}} \geq 0$, thus $K_{\lambda^{*}} \equiv 0$. This contradicts the assumption that $K_{\lambda^{*}}$ is not a constant.

Step 3. $u_{\lambda}$ is uniformly bounded in $W^{1,2}(V)$ as $\lambda \rightarrow \lambda^{*}$.

Fixing $x_{0} \in V_{\lambda^{*}}^{-}$, we set $\rho \in V^{\mathbb{R}}$ to be a function which vanishes besides $x_{0}$ and $\rho\left(x_{0}\right)<0$. Consider the equation

$$
\Delta w+\kappa-\rho e^{2 w}=0
$$

which always has the unique solution from the case (1) in Theorem 2 set $w_{1}$. As above, the function $v_{\lambda}=u_{\lambda}-w_{1}$ satisfies the equation

$$
\Delta v_{\lambda}+\rho e^{2 w_{1}}-K_{\lambda} e^{2\left(v_{\lambda}+w_{1}\right)}=0 .
$$

Multiplying (24) by $e^{2 v_{\lambda}}$ and integrating by parts over $V$ gives

$$
\int_{V} e^{2 v_{\lambda}} \Delta v_{\lambda} d \mu+\int_{V} \rho e^{2\left(v_{\lambda}+w_{1}\right)} d \mu-\int_{V} K_{\lambda} e^{2\left(2 v_{\lambda}+w_{1}\right)} d \mu=0 .
$$

Utilizing the fact that

$$
\left(e^{2 a}-e^{2 b}\right)(a-b) \geq\left(e^{a}-e^{b}\right)^{2}, \quad a, b \in \mathbb{R},
$$

one can estimate the first term in 25) as follows.

$$
\begin{aligned}
& \int_{V} e^{2 v_{\lambda}} \Delta v_{\lambda} d \mu=\frac{1}{2} \sum_{x, y \in V} \omega_{x y}\left(e^{2 v_{\lambda}(x)}-e^{2 v_{\lambda}(y)}\right)\left(v_{\lambda}(x)-v_{\lambda}(y)\right) \\
& \geq \frac{1}{2} \sum_{x, y \in V} \omega_{x y}\left(e^{v_{\lambda}(x)}-e^{v_{\lambda}(y)}\right)^{2} \\
&=\int_{V}\left|\nabla e^{v_{\lambda}}\right|^{2} d \mu \\
& 13
\end{aligned}
$$


Inserting this estimate into 25], we obtain

$$
\int_{V}\left|\nabla e^{v_{\lambda}}\right|^{2} d \mu+\int_{V} \rho e^{2\left(v_{\lambda}+w_{1}\right)} d \mu-\int_{V} K_{\lambda} e^{2\left(2 v_{\lambda}+w_{1}\right)} d \mu \leq 0 .
$$

On the other hand, let $h=e^{v_{\lambda}}$ in $(22)$, yields

$$
\int_{V}\left|\nabla e^{v_{\lambda}}\right|^{2} d \mu-2 \int_{V} K_{\lambda} e^{2\left(2 v_{\lambda}+w_{1}\right)} d \mu \geq 0 .
$$

Together with (26), we have

$$
\int_{V}\left|\nabla e^{v_{\lambda}}\right|^{2} d \mu \leq-2 \int_{V} \rho e^{2\left(v_{\lambda}+w_{1}\right)} d \mu=-2 \rho\left(x_{0}\right) e^{2 u_{\lambda}\left(x_{0}\right)} .
$$

One may derive that $u_{\lambda}\left(x_{0}\right)$ is the uniform bound, i.e. there exists a constant $C>0$ such that

$$
e^{2 u_{\lambda}\left(x_{0}\right)} \leq C
$$

Indeed, if $u_{\lambda}\left(x_{0}\right) \leq 0$, the above inequality is obvious; if $u_{\lambda}\left(x_{0}\right)>0$, as in the proof of Lemma 5 see (11), one can get the boundedness of $u_{\lambda}\left(x_{0}\right)$ as well. Together with (27), yields

$$
\int_{V}\left|\nabla e^{v_{\lambda}}\right|^{2} d \mu \leq C^{\prime}
$$

Next, we claim that $e^{v_{\lambda}}$ and thus $e^{u_{\lambda}}$ is uniformly bounded in $\ell_{\mu}^{2}$. Suppose not, we may assume that $\left\|e^{v_{\lambda}}\right\|_{\ell_{\mu}^{2}} \rightarrow \infty$ as $\lambda \rightarrow \lambda^{*}$. Let

$$
w_{\lambda}=\frac{e^{v_{\lambda}}}{\left\|e^{v_{\lambda}}\right\|_{\ell_{\mu}^{2}}}
$$

then $\left\|w_{\lambda}\right\|_{\ell_{\mu}^{2}}=1$, and $\left\|\nabla w_{\lambda}\right\|_{\ell_{\mu}^{2}} \rightarrow 0$ from (28). It follows that $w_{\lambda}$ converges to a constant in $W^{1,2}(V)$. From (3.3), we have $w_{\lambda}\left(x_{0}\right) \rightarrow 0$, and hence $w_{\lambda} \equiv 0$ on $V$, which contradicts $\left\|w_{\lambda}\right\|_{\ell_{\mu}^{2}}=1$ and then confirms our claim. Since $u_{\lambda}$ is bounded below by (23) in Step 1, one has the $\ell_{\mu^{-}}^{2}$ boundedness of $u_{\lambda}$, and thus the $W^{1,2}(V)$-boundedness of $u_{\lambda}$.

\subsection{No solution when $\lambda>\lambda^{*}$.}

Proof of this case is a consequence of the upper and lower solutions principle, as follows.

Proof of (4) in Theorem 2. Let $u_{\lambda_{1}}$ be the solution of (4) at some $\lambda_{1}>\lambda^{*}$. For any $0<\lambda<\lambda_{1}$, $u_{\lambda_{1}}$ is an upper solution of (4) at $\lambda$. Indeed,

$$
\Delta u_{\lambda_{1}}+\kappa-(K+\lambda) e^{2 u_{\lambda_{1}}}=\left(\lambda_{1}-\lambda\right) e^{2 u_{\lambda_{1}}}>0
$$

From (15), it is easy to get a lower bound solution $\varphi$ of (4) at $\lambda$ such that $\varphi \leq u_{\lambda_{1}}$. By the upper and lower solutions principle, there exists a solution of (4) at $\lambda$, which contradicts the definition of $\lambda^{*}$.

\section{References}




\section{References}

[1] A. Ambrosetti, P. Rabinowitz, Dual variational methods in critical point theory and applications, J. Funct. Anal. 14 (1973) 349-381.

[2] F. Borer, L. Galimberti, M. Struwe, "Large" conformal metrics of prescribing Gauss curvature on surfaces of high genus, Comment. Math. Helv. 90 (2015) 407-428.

[3] F. Camilli, C. Marchi, A note on Kazdan-Warner equation on networks, arXiv:1909.08472

[4] W. Ding, J. Liu, A note on the problem of prescribing Gaussian curvature on surfaces, Trans. Amer. Math. Soc. 347 (1995) 1059-1066.

[5] H. Ge, Kazdan-Warner equation on graph in the negative case, J. Math. Anal. Appl. 453 (2017) 1022-1027.

[6] H. Ge, W. Jiang, Kazdan-warner equation on infinite graphs, J. Korean Math. Soc. 55 (2018) 1091-1101.

[7] A. Grigor'yan, Y. Lin, Y. Yang, Kazdan-Warner equation on graph, Calc. Var. Partial Differential Equations 55 (2016), no. 4, Art. 92, 13 pp.

[8] A. Grigor'yan, Y. Lin, Y. Yang, Yamabe type equations on graphs. J. Differential Equations 261 (2016) 4924-4943.

[9] A. Grigor'yan, Y. Lin, Y. Yang, Existence of positive solutions to some nonlinear equations on locally finite graphs, Sci. China Math. 60 (2017) 1311-1324.

[10] X. Han, M. Sha, L. Zhao, Existence and convergence of solutions for nonlinear biharmonic equations on graphs, to appear in J. Differential Equations, https://doi.org/10.1016/j.jde.2019.10.007

[11] M. Keller, M. Schwarz, The Kazdan-Warner equation on canonically compactifiable graphs, Calc. Var. Partial Differential Equations 57 (2018), no. 2, Art. 70, 18 pp.

[12] J. Kazdan, F. Warner, Curvature functions for compact 2-manifolds, Ann. of Math. 99 (1974) 14-47.

[13] J. Kazdan, F. Warner, Curvature functions for open 2-manifolds, Ann. of Math. 99 (1974) 203-219.

[14] Y. Lin, Y. Wu, The existence and nonexistence of global solutions for a semilinear heat equation on graphs, Calc. Var. Partial Differential Equations 56 (2017), no. 4, Art. 102, 22 pp.

[15] M. Struwe, Critical points of embeddings of $H_{0}^{1, n}$ into Orlicz spaces, Ann. Inst. H. Poincaré Anal. Non Linéaire 5 (1988) 425-464.

[16] M. Struwe, The existence of surfaces of constant mean curvature with free boundaries, Acta Math. 160 (1988) 19-64.

[17] Y. Yang, X. Zhu, Prescribing Gaussian curvature on closed Riemann surface with conical singularity in the negative case, Ann. Acad. Sci. Fenn. Math. 44 (2019) 167-181. 